\title{
From the gut to the strut: where inflammation reigns, bone abstains
}

\author{
Jameel Iqbal, Tony Yuen, Li Sun, and Mone Zaidi \\ Mount Sinai Bone Program, Icahn School of Medicine at Mount Sinai, New York, New York, USA.
}

In this issue of the $J \mathrm{Cl}$, Li et al. show that germ-free mice, when chemically castrated, do not lose bone - a finding that unequivocally establishes a role of gut microbiota in mediating hypogonadal bone loss. Additionally and not unexpectedly, probiotics reversed hypogonadal osteopenia in sex steroid-deficient mice by preventing the disruption of gut barrier function and dampening cytokine-induced inflammation. The authors propose that TNF $\alpha$ is a key mediator; however, it is very likely that other molecules - including IL-1, IL-6, IL-17, RANKL, OPG, and CCL2 - modulate probiotic action. The results of this study highlight the potential for repurposing probiotics for the therapy of osteoporosis. Future placebo-controlled clinical trials will be required to establish safety and efficacy of probiotics in reducing fracture risk in people.

\section{A new player in sex steroid} deficiency-related bone loss

Osteoporosis is a leading cause of morbidity in the increasing population of aging adults. In postmenopausal women, fracture incidence far exceeds the combined incidence of breast cancer, stroke, and myocardial infarction. Bone loss arises from accelerated resorption by osteoclasts, which outpaces the accompanying increase in bone formation by osteoblasts (1). Postmenopausal osteoporosis has traditionally been solely attributed to declining estrogen levels. However, the rapid loss of bone during the late perimenopausal transition, particularly when estrogen levels are relatively normal, is proposed to be mediated, at least in part, by rising follicle-stimulating hormone levels (FSH levels) $(2,3)$. Nonetheless, these hormonal changes do not fully explain the increased bone formation, high BM T cell counts, or macrophage activation that have been noted across the menopausal transition (4). Alterations in immune cell function have largely been attributed to increased production of $\mathrm{TNF} \alpha$, which further enhances osteoclast formation and function $(5,6)$. Consistent with this, ablation of the Tnfa gene in mice prevents gonadectomy-induced bone loss, osteoclast and osteoblast activation, and accompanying immune cell abberations (5). However, the mechanisms that drive TNF $\alpha$ production during hypogonadal states remain relatively unclear. In this issue, Li et al. (7) show that gut microbiota play a fundamental role in the enhanced $\mathrm{TNF} \alpha$ production that occurs upon the induction of estrogen deficiency in mice.

It has previously been shown that lowering the inflammatory burden does suppress the effects of estrogen deficiency. For example, TNF $\alpha$ or IL-1 blockade in early postmenopausal women decreases bone resorption markers (8). Likewise, mice in which Tnfa or Il6 is deleted are relatively resistant to ovariectomy-induced bone loss (9). Li et al. evaluated how gut

Related Article: p. 2049

Conflict of interest: M. Zaidi is/has been a consultant for Merck, Novartis, Roche, Gerson Lehmann Group, and Guidepoint. He will be entitled to proceeds from any licensing agreement between Icahn School of Medicine at Mount Sinai and any commercial entity for a US patent relating to the use of follicle-stimulating hormone (FSH) blockers in preventing bone loss.

Reference information: / Clin Invest. 2016;126(6):2045-2048. doi:10.1172/JCI87430. 
A

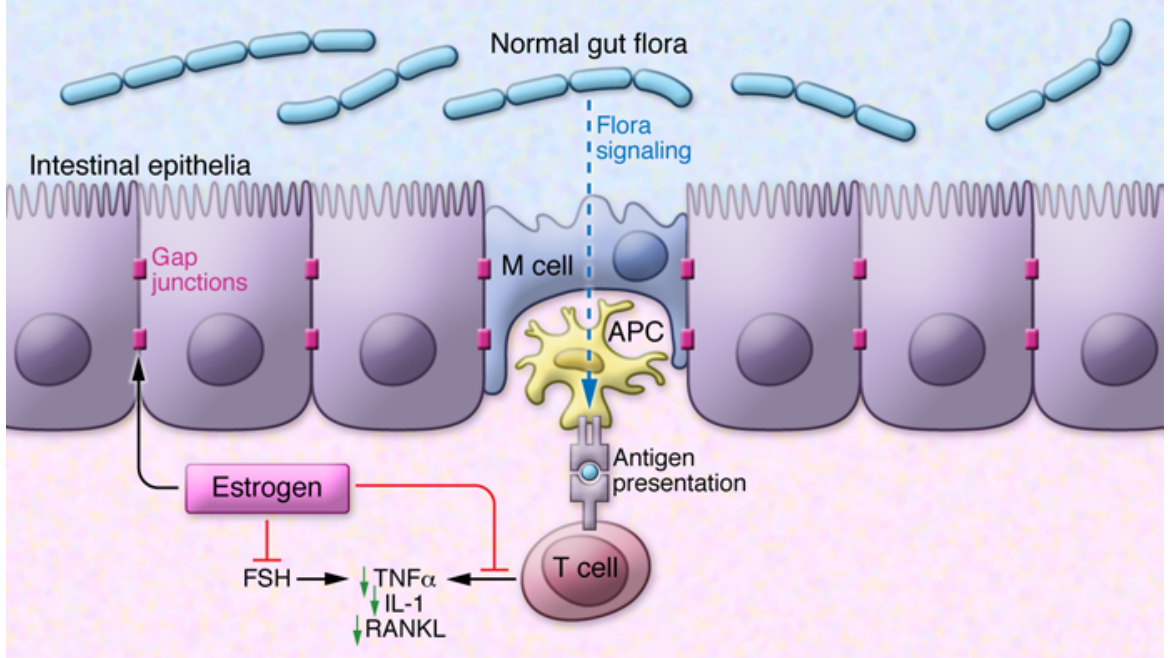

B

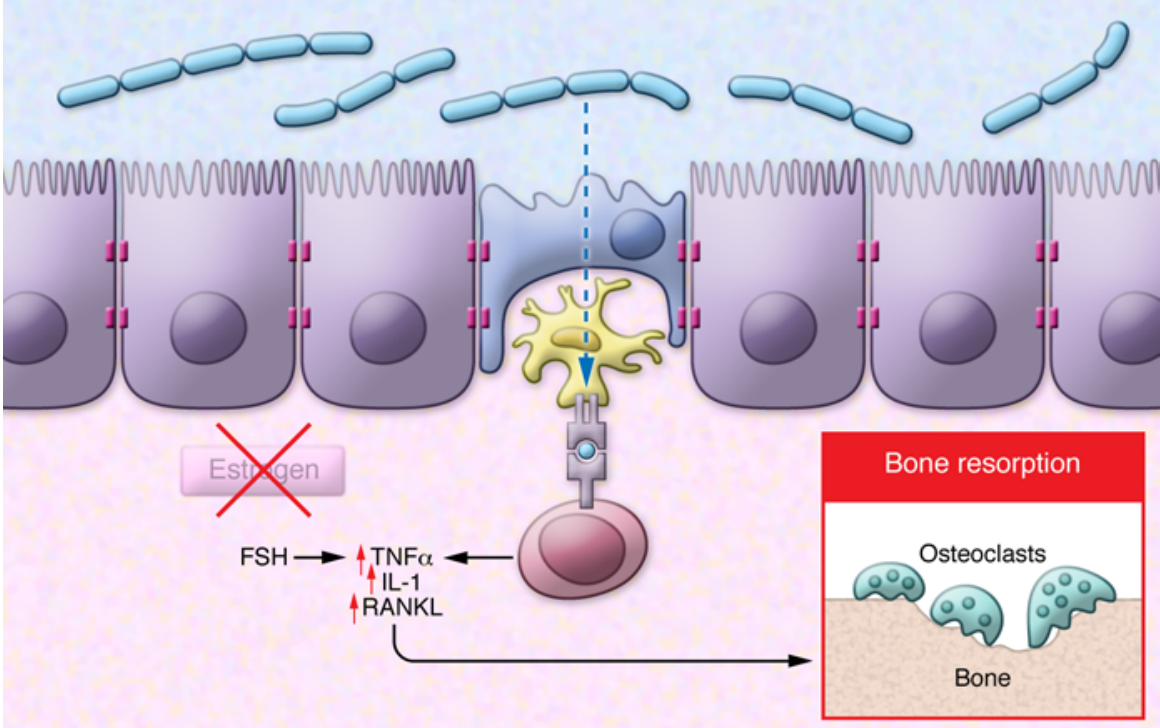

C

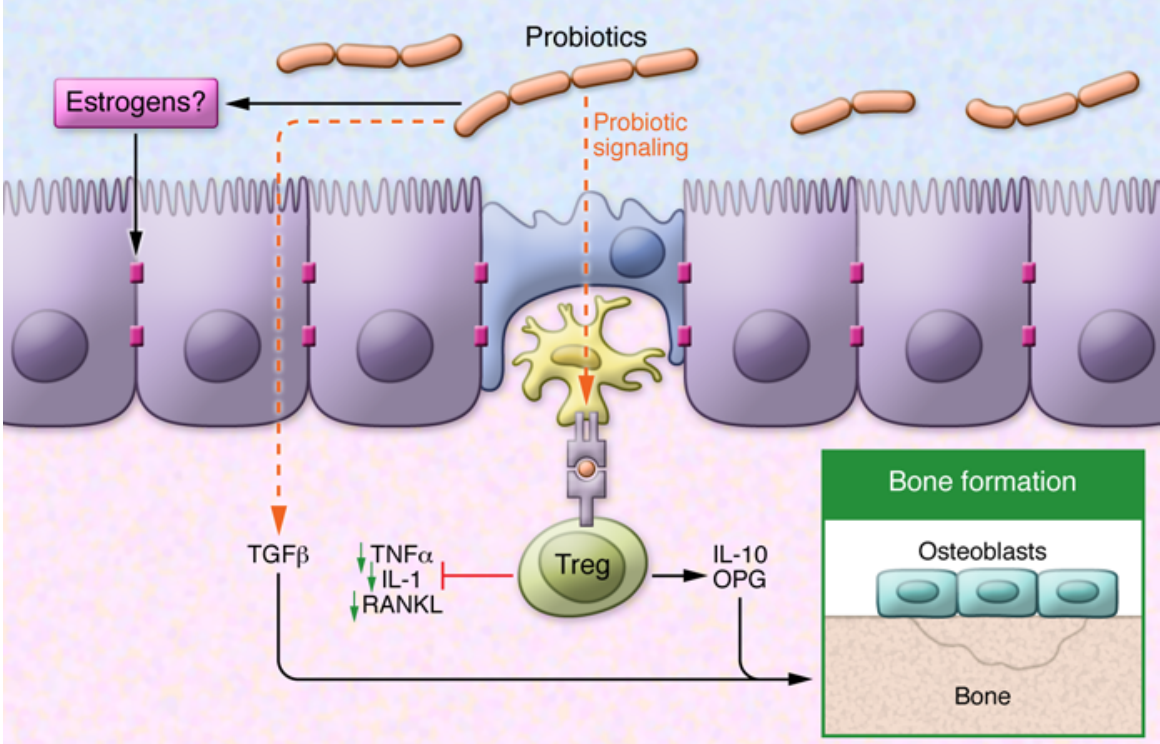

Figure 1. Gut microbiota play a fundamental role in bone mass regulation. (A) Normal gut flora in the face of hypogonadism cause a proinflammatory immune response, leading to enhanced production of TNF $\alpha$, IL-1 $\beta$, RANKL, and CCL2 - among other cytokines and chemokines - from T cells and phagocytes. (B) These molecules in turn drive osteoclastic bone resorption and decrease bone mass. Estrogen serves to dampen this proinflammatory cascade via the gut through several mechanisms. It augments gap junction and cell-to-cell contacts, thus preventing the microbiota from inducing inflammation. It also directly suppresses proinflammatory $T$ cell production and, indirectly, lowers FSH levels, thus attenuating FSH-induced TNF $\alpha$ production (6). (C) Probiotics decrease TNF $\alpha$ and $\mathrm{IL}-1 \beta$ levels and increase the production of IL-10 and OPG. These effects are mediated, in part, through an increase in the number of Tregs and increased TCF $\beta 1$, which together can also enhance bone formation. An additional mechanism involves the secretion of estrogen-like compounds from probiotics. These compounds likely recapitulate many of the antiinflammatory actions of endogenous estrogen, such as augmenting epithelial cell contacts.

inflammation and $\mathrm{T}$ cell activation. Consistent with this, Li et al. show that estrogen deficiency causes the loss of barrier function, leading to endotoxemia (Figure 1, ref. 7). In contrast, germ-free mice maintained adequate barrier function following estrogen deficiency, an observation that is in concordance with previous studies showing low $\mathrm{CD} 4^{+} \mathrm{T}$ cell counts and TNF $\alpha$ levels in these mice (10). Therefore, inflammation induced by sex steroid deficiency in a setting of normal microbiota should lead to $\mathrm{T}$ cell activation, which in turn would be expected to increase the production of proinflammatory cytokines, including TNF $\alpha$ (14). That TNF $\alpha$ can itself cause bone loss by increasing osteoclastic bone resorption has been proven unequivocally in Tnfa-expressing transgenic mice. Now, Li et al. have established a permissive connection between sex steroid deficiency, gut microbiota, inflammation, and TNF $\alpha$ production by showing that hypogonadal germ-free mice are unable to increase $\mathrm{TNF} \alpha$-expressing $\mathrm{CD} 4^{+} \mathrm{T}$ cells.

\section{The benefits of probiotics}

The identification of a critical role for gut microbiota in mediating the bone loss of sex steroid deficiency begs the question as to whether this pathway can be utilized to 
provide a therapeutic advantage for osteoporosis patients. Exposure of weaning mice to antibiotics increases bone mass (15), suggesting that alteration of the normal microbiota may, in fact, recapitulate the beneficial skeletal actions of a germ-free environment. An alternative strategy in lieu of antibiotics would be to use probiotics live microorganisms that provide health benefits to the host (16). Probiotics have been long known to increase bone strength in chickens (17) and to improve eggshell hardness (16). Several other notable observations underscore the premise that altering the microbiota using probiotics can alter bone remodeling and/or bone mass in hypogonadal states. First, while estrogen deficiency impairs calcium absorption, probiotics reverse this effect and suppress bone resorption (18). Second, the probiotic bifidobacterium blunts the decreases in bone mass and increases in osteoclast numbers in hypogonadal rats (19). Li et al. have further solidified these observations by showing that two different probiotic classes are able to prevent the bone loss that is secondary to estrogen deficiency (7). However, probiotics also independently caused an increase in bone mass in sham controls without hypogonadal hyperresorption, suggesting a possible enhancement of osteoblastic bone formation.

These beneficial effects of probiotics on bone formation lead to a further question: can probiotics reverse hypogonadal bone loss by decreasing proinflammatory cytokines, including TNF $\alpha$ and IL-1 $\beta$, and/ or by increasing antiinflammatory cytokines such as IL-10 (20)? Several lines of evidence attest to this being the case, at least in part. Probiotics do decrease TNF $\alpha$ and IL-1 $\beta$ levels and increase production of the antiosteoclastogenic cytokine osteoprotegerin (OPG) in hypogonadal mice (21). They also inhibit Tnfa and Il6 expression and upregulate $I l 1 O$ in a collagen-induced arthritis model (22) - effects that are likely exerted via NFкB dephosphorylation (23). Importantly, media from the probiotic Lactobacillus is able to decrease both macrophage Tnfa expression and osteoclastogenesis $(24,25)$. Consistent with these findings, Li et al. note that probiotic-fed hypogonadal mice display significant decrements in Tnfa and Rankl in both the intestine and BM (7). Furthermore, probiotics decreased gut permeability by activating the same MAP kinases that are induced nongenomically by estrogen. This action likely results from the production of small estrogen-like molecules that may behave as selective estrogen receptor modulators with differential actions on bone. It is therefore not surprising that probiotics display sex-specific actions on bone mass in mice (26).

\section{Conclusions}

If TNF $\alpha$ inhibition is indeed central to the action of probiotics in preventing hypogonadal bone loss, sequestration of $\mathrm{TNF} \alpha$ by the monoclonal antibody infliximab in people - for example, those suffering from inflammatory bowel disease - would be expected to reduce bone loss. Surprisingly, such an effect was not found in a cohort of patients with Crohn's disease (27), leaving the question open as to whether TNF $\alpha$ is the sole mediator of probiotic action on bone. In fact, multiple studies underscore the complexity of TNF $\alpha$ action in terms of the multiplicity of target genes that it modulates, as well as the temporal oscillations in gene expression that it elicits $(28,29)$. More directly, transcriptional profiling of the peripheral blood from people fed Lactobacillus has not only been shown to reduce expression of multiple $\mathrm{NF \kappa B}$-responsive genes, but also to inhibit the osteoclastogenic chemokine CCL2 (30). This makes CCL2 and its receptor CCR2 potential mediators of probiotic action, particularly as the genetic deletion of either leads to reduced osteoclastogenesis (31). Additionally, IL-17, which has been recently identified as a modulator of hypogonadal bone loss, is reduced by probiotic administration. In contrast, probiotics increase TGF $\beta 1$ to enhance Tregs in murine models of inflammatory disease (32) and therefore prevent ovariectomy-induced Treg downregulation (21). As Tregs inhibit osteoclast differentiation, this action is likely to be osteoprotective (33). It is therefore possible that secreted molecules other than TNF $\alpha$ may play critical roles in mediating the effects of probiotics on bone.

\section{Acknowledgments}

M. Zaidi acknowledges the NIH for support (AG40132, AG23176, AR65932, and AR67066).

Address correspondence to: Mone Zaidi, Endocrinology, PO 1055, Icahn School of Medicine at Mount Sinai, One Gustave L.
Levy Place, New York, New York 10029, USA. Phone: 212.241.8797; E-mail: Mone.zaidi@mssm.edu.

1. Zaidi M. Skeletal remodeling in health and disease. Nat Med. 2007;13(7):791-801.

2. Randolph JF Jr, Sowers M, Bondarenko IV, Harlow SD, Luborsky JL, Little RJ. Change in estradiol and follicle-stimulating hormone across the early menopausal transition: effects of ethnicity and age. JClin Endocrinol Metab. 2004;89(4):1555-1561.

3. Sun L, et al. FSH directly regulates bone mass. Cell. 2006;125(2):247-260.

4. Iqbal J, Zaidi M. Understanding estrogen action during menopause. Endocrinology. 2009;150(8):3443-3445.

5. Roggia C, et al. Up-regulation of TNF-producing $\mathrm{T}$ cells in the bone marrow: a key mechanism by which estrogen deficiency induces bone loss in vivo. Proc Natl Acad Sci U S A. 2001;98(24):13960-13965.

6. Iqbal J, Sun L, Kumar TR, Blair HC, Zaidi M. Follicle-stimulating hormone stimulates TNF production from immune cells to enhance osteoblast and osteoclast formation. Proc Natl Acad Sci U S A. 2006;103(40):14925-14930.

7. Li JY, et al. Sex steroid deficiency induces bone loss in a gut microbiota dependent manner. JClin Invest. 2016;126(6):2049-2063.

8. Charatcharoenwitthaya N, Khosla S, Atkinson EJ, McCready LK, Riggs BL. Effect of blockade of TNF- $\alpha$ and interleukin- 1 action on bone resorption in early postmenopausal women. J Bone Miner Res. 2007;22(5):724-729.

9. Manolagas SC, Jilka RL. Bone marrow, cytokines, and bone remodeling. Emerging insights into the pathophysiology of osteoporosis. N Engl JMed. 1995;332(5):305-311.

10. Sjogren K, et al. The gut microbiota regulates bone mass in mice. J Bone Miner Res. 2012;27(6):1357-1367.

11. Kong YY, et al. Activated T cells regulate bone loss and joint destruction in adjuvant arthritis through osteoprotegerin ligand. Nature. 1999;402(6759):304-309.

12. Mistry PK, et al. Glucocerebrosidase genedeficient mouse recapitulates Gaucher disease displaying cellular and molecular dysregulation beyond the macrophage. Proc Natl Acad SciU S A. 2010;107(45):19473-19478.

13. Liu J, et al. Gaucher disease gene GBA functions in immune regulation. Proc Natl Acad Sci U S A. 2012;109(25):10018-10023.

14. Weitzmann MN, Pacifici R. T cells: unexpected players in the bone loss induced by estrogen deficiency and in basal bone homeostasis. Ann N Y Acad Sci. 2007;1116:360-375.

15. Cho I, et al. Antibiotics in early life alter the murine colonic microbiome and adiposity. Nature. 2012;488(7413):621-626.

16. McCabe L, Britton RA, Parameswaran N. Prebiotic and probiotic regulation of bone health: role of the intestine and its microbiome. Curr Osteoporos Rep. 2015;13(6):363-371.

17. Mutus R, Kocabagli N, Alp M, Acar N, Eren $\mathrm{M}$, Gezen SS. The effect of dietary probiotic 
supplementation on tibial bone characteristics and strength in broilers. Poult Sci. 2006;85(9):1621-1625.

18. Zafar TA, Weaver CM, Zhao Y, Martin BR, Wastney ME. Nondigestible oligosaccharides increase calcium absorption and suppress bone resorption in ovariectomized rats. J Nutr. 2004;134(2):399-402.

19. Parvaneh K, et al. Probiotics (Bifidobacterium longum) increase bone mass density and upregulate Sparc and Bmp-2 genes in rats with bone loss resulting from ovariectomy. Biomed Res Int. 2015;2015:897639.

20. Garcia VG, et al. Effect of the probiotic Saccharomyces cerevisiae on ligature-induced periodontitis in rats. J Periodontal Res. 2016;51(1):26-37.

21. Ohlsson C, et al. Probiotics protect mice from ovariectomy-induced cortical bone loss. PLoS One. 2014;9(3):e92368.

22. Amdekar S, Singh V, Singh R, Sharma P, Keshav P, Kumar A. Lactobacillus casei reduces the inflammatory joint damage associated with collagen-induced arthritis (CIA) by reducing the pro-inflammatory cytokines: Lactobacillus casei: COX-2 inhibitor. J Clin Immunol.
2011;31(2):147-154.

23. Ammoscato F, et al. Lactobacillus rhamnosus protects human colonic muscle from pathogen lipopolysaccharide-induced damage. Neurogastroenterol Motil. 2013;25(12):984-e777.

24. Fong FL, Kirjavainen PV, El-Nezami H. Immunomodulation of Lactobacillus rhamnosus GG (LGG)-derived soluble factors on antigenpresenting cells of healthy blood donors. Sci Rep. 2016;6:22845.

25. Britton RA, et al. Probiotic L. reuteri treatment prevents bone loss in a menopausal ovariectomized mouse model. JCell Physiol. 2014;229(11):1822-1830.

26. McCabe LR, Irwin R, Schaefer L, Britton RA. Probiotic use decreases intestinal inflammation and increases bone density in healthy male but not female mice. J Cell Physiol. 2013;228(8):1793-1798

27. Pazianas M, Rhim AD, Weinberg AM, Su C, Lichtenstein GR. The effect of anti-TNF-alpha therapy on spinal bone mineral density in patients with Crohn's disease. Ann N Y Acad Sci. 2006;1068:543-556.

28. Sun L, Yang G, Zaidi M, Iqbal J. TNF-induced oscillations in combinatorial transcription factor binding. Biochem Biophys Res Commun 2008;371(4):912-916.

29. Sun L, Yang G, Zaidi M, Iqbal J. TNF-induced gene expression oscillates in time. Biochem Biophys Res Commun. 2008;371(4):900-905.

30. Solano-Aguilar G, et al. Dawson H, Andreyeva I, et al. Transcriptomic profile of whole blood cells from elderly subjects fed probiotic bacteria Lactobacillus rhamnosus GG ATCC 53103 (LGG) in a Phase I Open Label Study. PLoS One. 2016;11(2):e0147426.

31. Khan UA, Hashimi SM, Bakr MM, Forwood MR, Morrison NA. CCL2 and CCR2 are essential for the formation of osteoclasts and foreign body giant cells. J Cell Biochem. 2016;117(2):382-389.

32. Kwon HK, et al. Generation of regulatory dendritic cells and $\mathrm{CD} 4^{+}{ }^{-}$oxp $3^{+} \mathrm{T}$ cells by probiotics administration suppresses immune disorders. Proc Natl Acad Sci U S A. 2010;107(5):2159-2164.

33. Kim YG, Lee CK, Nah SS, Mun SH, Yoo B, Moon HB. Human $\mathrm{CD} 4^{+} \mathrm{CD} 25^{+}$regulatory T cells inhibit the differentiation of osteoclasts from peripheral blood mononuclear cells. Biochem Biophys Res Commun. 2007;357(4):1046-1052. 